\title{
Particle Orbit Analysis in the Finite Beta Plasma of the Large Helical Device using Real Coordinates
}

\author{
Ryousuke SEKI，Yutaka MATSUMOTO, Yasuhiro SUZUKI ${ }^{1)}$, Kiyomasa WATANABE ${ }^{1)}$ \\ and Masafumi ITAGAKI \\ Graduate School of Engineering, Hokkaido University, Sapporo 060-8628, Japan \\ ${ }^{1)}$ National Institute for Fusion Science, Toki 509-5292, Japan
}

(Received 6 December 2007 / Accepted 15 February 2008)

\begin{abstract}
High-energy particles in a finite beta plasma of the Large Helical Device (LHD) are numerically traced in a real coordinate system. We investigate particle orbits by changing the beta value and/or the magnetic field strength. No significant difference is found in the particle orbit classifications between the vacuum magnetic field and the finite beta plasma cases. The deviation of a banana orbit from the flux surfaces strongly depends on the beta value, although the deviation of the orbit of a passing particle is independent of the beta value. In addition, the deviation of the orbit of the passing particle, rather than that of the banana-orbit particles, depends on the magnetic field strength. We also examine the effect of re-entering particles, which repeatedly pass in and out of the last closed flux surface, in the finite beta plasma of the LHD. It is found that the number of re-entering particles in the finite beta plasma is larger than that in the vacuum magnetic field. As a result, the role of reentering particles in the finite beta plasma of the LHD is more important than that in the vacuum magnetic field, and the effect of the charge-exchange reaction on particle confinement in the finite beta plasma is large.
\end{abstract}

(c) 2008 The Japan Society of Plasma Science and Nuclear Fusion Research

Keywords: finite beta, re-entering particle, LHD, particle orbit

DOI: $10.1585 /$ pfr.3.016

\section{Introduction}

The Large Helical Device (LHD) is a heliotron-type device with $\ell=2 / m=10, A_{\mathrm{p}}=5.8-8.3, B_{\mathrm{ax}}$ up to $3 \mathrm{~T}$, and a helical divertor [1]. The typical plasma major radius $R_{\mathrm{ax}}$ and the averaged plasma minor radius $a_{\mathrm{p}}$ in vacuum are 3.6 and $0.64 \mathrm{~m}$, respectively. Here, $\ell$ and $m$ are a poloidal and toroidal period number of the helical coils, $A_{\mathrm{p}}$ is a plasma aspect ratio, and $B_{\mathrm{ax}}$ is the magnetic field strength at $R_{\mathrm{ax}}$.

In recent experiments using the LHD, the volumeaveraged beta value $\left\langle\beta_{\mathrm{dia}}\right\rangle$ reached $4.5 \%$ by neutral beam injection (NBI) heating [2]. As is well known, an equilibrium magnetic field in a finite beta plasma of the LHD differs significantly from a vacuum magnetic field (Fig. 1). The magnetic axis shifts the torus outward because of the Shafranov shift. In addition, the flux surfaces in the periphery of the plasma are destroyed by the finite beta effect [3]. As a result, the volume of the last closed flux surface (LCFS) becomes small.

Effective plasma heating by high-energy particles produced by NBI is required in order to achieve a high beta value. Therefore, the behavior of high-energy particles in a finite beta plasma is important in studies on plasma heating. In particular, it is of great importance in heliotorontype devices such as the LHD, since the confinement of high-energy particles in heliotron devices has been believed to be poor. The behavior of high-energy particles

author'se-mail: seki@fusion.qe.eng.hokudai.ac.jp in the finite beta plasmas of the LHD has been studied using the Boozer coordinate system $[4,5]$. Although the flux surfaces in the periphery of the finite beta plasma are supposed to be destroyed, closed flux surfaces are assumed in the Boozer coordinates. Therefore, high-energy particles in the finite beta plasmas cannot be accurately traced in the Boozer coordinates. Moreover, in studies using the Boozer coordinates, the particles passing out of the LCFS have been regarded as lost particles since the particle-loss boundary has usually been set on the LCFS.

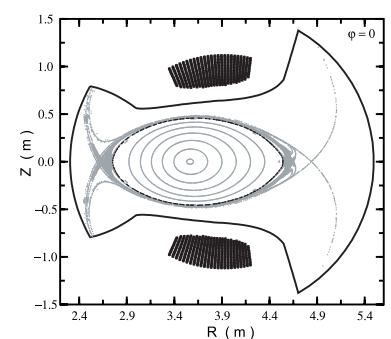

(a) vacuum magnetic field $(\langle\beta\rangle=0 \%)$

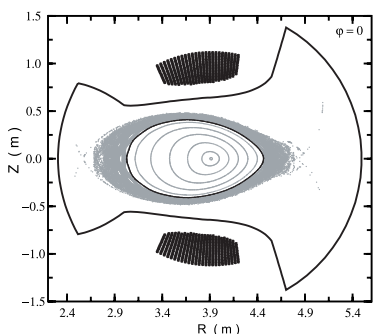

(b) finite beta plasma $(\langle\beta\rangle=2.7 \%)$
Fig. 1 Poincaré plots of the magnetic field lines on the horizontally elongated poloidal plane in the LHD in the case of (a) vacuum magnetic field $(\langle\beta\rangle=0 \%)$ and (b) finite beta plasma $(\langle\beta\rangle=2.7 \%)$. Black points denote the LCFS and gray points denote the magnetic field lines. The vacuum vessel wall and helical coils of the LHD are also shown. 
On the other hand, particle orbit analyses, in which the particle-loss boundary is set on the vacuum vessel wall, have been performed. In these analyses, it has been shown that re-entering particles, which repeatedly pass in and out of the LCFS, exist in the vacuum magnetic field and that they are confined within the vacuum vessel wall for a long time [6-8]. In particle orbit analysis using the Boozer coordinates, the number of lost particles could be overestimated because the re-entering particles are usually regarded as lost particles.

We numerically traced high-energy particles in the finite beta plasma of the LHD with appropriately treating the re-entering particles. We investigate the high-energy particle orbits in the finite beta plasma by comparing them with those in the vacuum magnetic field. The high-beta experiments in the LHD have been conducted at $B_{\mathrm{ax}} \simeq 0.5 \mathrm{~T}$ [2]. We also investigate the particle orbits in the finite beta plasma for the case of $B_{\mathrm{ax}}=0.5 \mathrm{~T}$. The number of re-entering particles in the finite beta plasmas might be larger than that in the vacuum magnetic field, since the volume of the LCFS in the finite beta plasmas becomes small. Therefore, the role of re-entering particles in the finite beta plasma might be more important than that in the vacuum magnetic field. We examine the effect of the reentering particles on particle confinement in the finite beta plasma of the LHD. The re-entering particles might be lost because of a charge-exchange reaction with the neutral particles, since the re-entering particles pass through the peripheral region, in which the neutral particle density is higher than that in the core. Thus, we study the effect of the charge-exchange reaction on the re-entering particles.

In Sec. 2, the numerical model and initial conditions used for our calculations are described. The results of these calculations are summarized in Sec. 3. Section 4 is devoted to the discussion. the conclusion is provided in Sec. 5 .

\section{Method}

In order to trace the particles, we use the equilibrium magnetic fields obtained by the three-dimensional magnetohydrodynamic (MHD) equilibrium code, HINT [9, 10], in which the existence of nested flux surfaces is not assumed. The equilibrium magnetic fields used in our calculations are (i) $B_{\mathrm{ax}}=3 \mathrm{~T},\langle\beta\rangle=0 \%$, and $R_{\mathrm{ax}}=3.6 \mathrm{~m}$, (ii) $B_{\mathrm{ax}}=3 \mathrm{~T},\langle\beta\rangle=2.7 \%$, and $R_{\mathrm{ax}} \simeq 3.9 \mathrm{~m}$, and (iii) $B_{\mathrm{ax}}=0.5 \mathrm{~T},\langle\beta\rangle=2.7 \%$, and $R_{\mathrm{ax}} \simeq 3.9 \mathrm{~m}$. As mentioned above, the equilibrium magnetic field differs significantly from the vacuum magnetic field as the beta value increases. For example, the magnetic axis shifts from $R_{\mathrm{ax}}=3.6 \mathrm{~m}(\langle\beta\rangle=0 \%)$ to $R_{\mathrm{ax}} \simeq 3.9 \mathrm{~m}(\langle\beta\rangle=2.7 \%)$. In addition, the position of the LCFS at the inner side of the torus changes from $R \simeq 2.76 \mathrm{~m}(\langle\beta\rangle=0 \%)$ to $R \simeq 3.03 \mathrm{~m}$ $(\langle\beta\rangle=2.7 \%)$ on the horizontally elongated poloidal plane. In contrast, the position of the LCFS at the outer side of the torus rarely changes.

We trace the particles by numerically solving the guiding-center equations in a collisionless case. In order to trace the re-entering particles appropriately, the particleloss boundary must be set on the vacuum vessel wall, i.e., the particles reaching the vacuum vessel wall are regarded as the lost particles. Therefore, the rotating helical coordinate system [11] is adopted. We use the 6th-order Runge-Kutta formulas [12] and three-dimensional higherorder spline function [13] to accurately trace the complicated orbits of the particles in the plasma periphery.

The initial conditions are determined as follows. The traced particle is a proton, whose initial energy is assumed to be $100 \mathrm{keV}$ with reference to the energy of the NBI in the LHD $(180 \mathrm{keV}, 50 \mathrm{keV})$. The starting points of the protons are set on the horizontally elongated poloidal plane as

$$
\begin{aligned}
& R=2.65+0.05 \mathrm{n}_{R}\left(\mathrm{n}_{R}=0,1, \cdots, 45\right) \mathrm{m} \\
& Z=0 \\
& \phi=0
\end{aligned}
$$

where $(R, Z, \phi)$ are the cylindrical coordinates; $R$ is the major radius, and $\phi$ is the toroidal angle. The initial pitch angles $\chi_{0}$ are varied from $0.05 \pi$ to $0.95 \pi$ with a step size of $0.05 \pi$. The protons with these initial conditions are traced for a period of $30 \mathrm{~ms}$. Note that the energy relaxation time for a $100 \mathrm{keV}$ proton in the plasma, whose temperature is $3 \mathrm{keV}$ and density is $10^{20} \mathrm{~m}^{-3}$, is $27 \mathrm{~ms}$.

\section{Results}

Based on the results of particle orbit tracing, we classify the particles in the LHD into three groups: passing particles, banana-orbit particles, and chaotic-orbit particles [14]. The passing particles are not trapped by either the helical or toroidal ripples and move approximately along the magnetic field lines. The banana-orbit particles are continuously trapped by the helical ripple and rotate in the poloidal direction. The chaotic-orbit particles repeatedly transit between the localized orbit in the inner side of the torus and the blocked orbit [15] in the outer side of the torus. Figure 2 shows the typical orbits of three groups in magnetic field (i) $\left(B_{\mathrm{ax}}=3 \mathrm{~T},\langle\beta\rangle=0 \%\right.$, and $R_{\mathrm{ax}}=3.6 \mathrm{~m}$ ). The left-hand sides in Fig. 2 are Poincaré plots of the particles on the horizontally elongated poloidal plane. The right-hand sides in Fig. 2 are particle orbit projections on the poloidal plane in $(\psi-\theta)$ coordinates, where $\psi$ is the magnetic flux, and $\theta$ is the poloidal angle. Both the passing and banana-orbit particles form closed drift surfaces (Figs. 2 (a), (b)). However, the passing and banana-orbit particles are lost when they drift outside the stochastic (chaotic field line) region [16]. The chaotic-orbit particles repeatedly transit between the localized orbit and the blocked orbit and do not form closed drift surfaces [15] (Fig. 2 (c)). The particle orbits in the finite beta plasma of the LHD are studied based on this classification. 

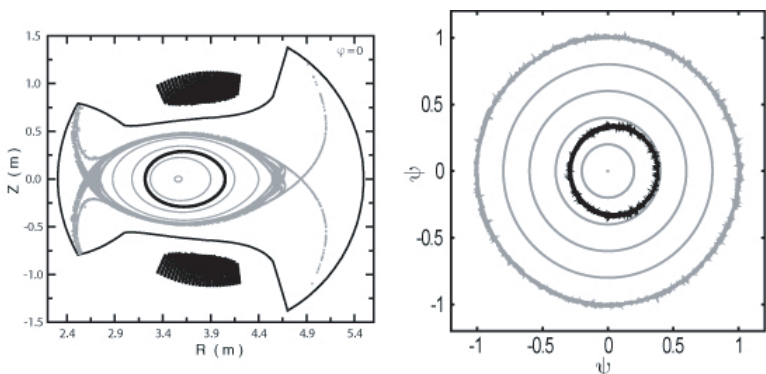

(a) passing particle $\left(\chi_{0}=0.1 \pi\right)$
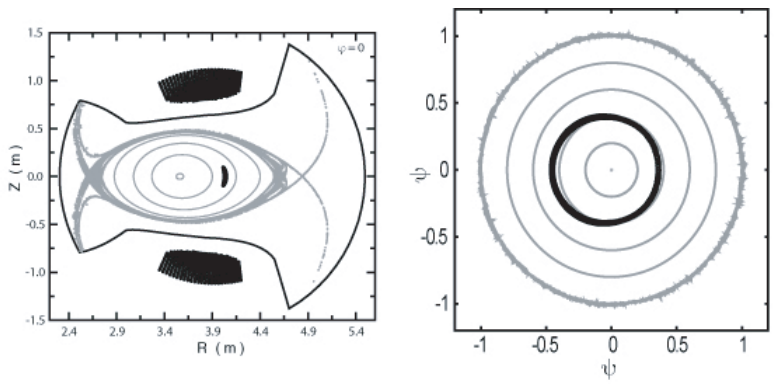

(b) banana-orbit particle $\left(\chi_{0}=0.55 \pi\right)$
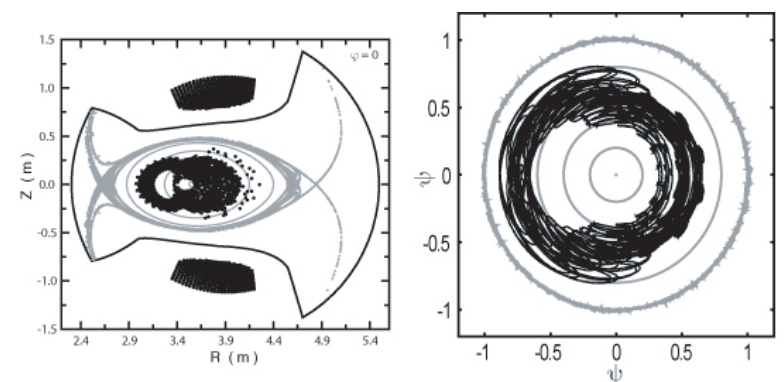

(c) chaotic-orbit particle $\left(\chi_{0}=0.65 \pi\right)$

Fig. 2 Typical orbits of the particles in magnetic field (i) $\left(B_{\mathrm{ax}}=\right.$ $3 \mathrm{~T},\langle\beta\rangle=0 \%$, and $R_{\mathrm{ax}}=3.6 \mathrm{~m}$ ). (a) Passing, (b) bananaorbit, and (c) chaotic-orbit particles are traced from $R=$ $4.05 \mathrm{~m}, Z=0$, and $\phi=0$. The left-hand sides of (a), (b), and (c) are the Poincaré plots of the particles on the horizontally elongated poloidal plane. The right-hand sides of (a), (b), and (c) are the particle orbit projections on the poloidal plane in $(\psi-\theta)$ coordinates, where $\psi$ is the magnetic fluxs, and $\theta$ is the poloidal angle.

\subsection{Magnetic field (i) $\left(B_{\mathrm{ax}}=3 \mathrm{~T},\langle\beta\rangle=0 \%\right.$, and $R_{\mathrm{ax}}=3.6 \mathrm{~m}$ )}

We compare the particle orbits in the finite beta plasma $\langle\beta\rangle=2.7 \%$ with those in the vacuum magnetic field $\langle\beta\rangle=0 \%$ in order to investigate the finite beta effects on the particle orbits. Figure 3 shows the particle orbit classifications in magnetic field (i) $\left(B_{\mathrm{ax}}=3 \mathrm{~T},\langle\beta\rangle=0 \%\right.$, and $R_{\mathrm{ax}}=3.6 \mathrm{~m}$ ) in the space relating to the starting points versus the initial pitch angles. The horizontal axis is the major radius of the starting points set on the line of $Z=0$ on the horizontally elongated poloidal plane. The vertical axis denotes the initial pitch angles divided by $\pi$. The po-

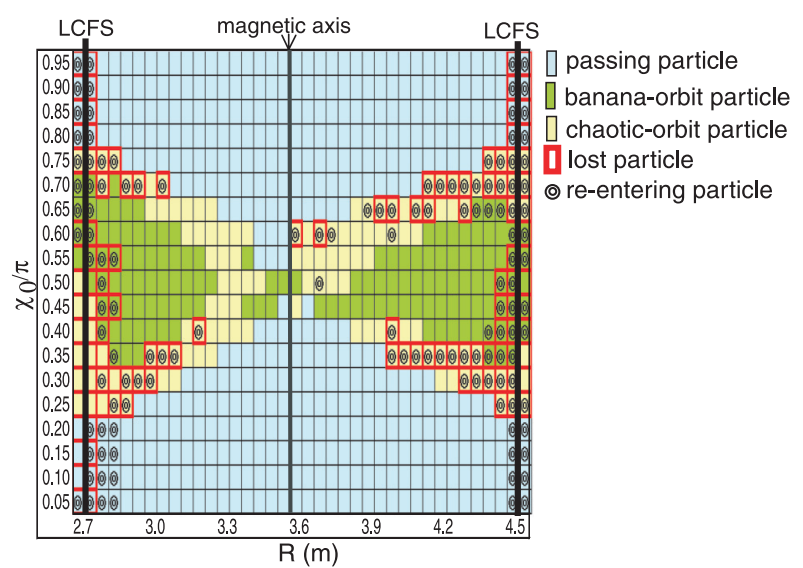

Fig. 3 Particle orbit classifications in magnetic field (i) $\left(B_{\mathrm{ax}}=\right.$ $3 \mathrm{~T},\langle\beta\rangle=0 \%$, and $R_{\mathrm{ax}}=3.6 \mathrm{~m}$ ) in the space relating to the starting points versus the initial pitch angles. The horizontal axis is the major radius $(R)$ of the starting points set on the line of $Z=0$ on the horizontally elongated poloidal plane. The vertical axis denotes the initial pitch angles $\left(\chi_{0}\right)$ divided by $\pi$. The red squares show the lost particles, i.e., the particles reaching the vacuum vessel wall within $30 \mathrm{~ms}$. The positions of the magnetic axis and the LCFS on the line of $Z=0$ on the horizontally elongated poloidal plane are also shown.

sitions of the magnetic axis and the LCFS on the line of $Z=0$ on the horizontally elongated poloidal plane are also shown. The red squares denote the lost particles, i.e., the particles reaching the vacuum vessel wall within $30 \mathrm{~ms}$.

Almost all the particles traced from near the magnetic axis become passing particles independent of the initial pitch angles. As the starting points are close to the LCFS, the range of the initial pitch angles that define the particles as passing particles becomes narrow. In the particles from near the LCFS, only the particles with $\chi_{0} \simeq 0$ or $\chi_{0} \simeq \pi$ become passing particles. On the other hand, particles with $\chi_{0} \simeq \pi / 2$ become banana-orbit particles. As the starting points are close to the LCFS, the range of the initial pitch angles that define the particles as the banana-orbit particles becomes wide. In the space relating to the starting points versus the initial pitch angles, the chaotic-orbit particles exist in the region almost surrounding the banana-orbit particles. However, some chaotic-orbit particles traced from $3.1 \mathrm{~m} \leq R \leq 4.3 \mathrm{~m}$ become lost particles.

The re-entering particles exist as particles traced from near the LCFS independent of particle orbit classifications. On the other hand, the re-entering particles traced from $3.1 \mathrm{~m} \leq R \leq 4.3 \mathrm{~m}$ are only chaotic-orbit particles.

\subsection{Magnetic field (ii) $\left(B_{\mathrm{ax}}=3 \mathrm{~T},\langle\beta\rangle=\right.$ $2.7 \%$, and $R_{\mathrm{ax}} \simeq 3.9 \mathrm{~m}$ )}

Figure 4 shows the particle orbit classifications in magnetic field (ii) $\left(B_{\mathrm{ax}}=3 \mathrm{~T},\langle\beta\rangle=2.7 \%\right.$, and $R_{\mathrm{ax}} \simeq$ $3.9 \mathrm{~m}$ ) in the space relating to the starting points versus 


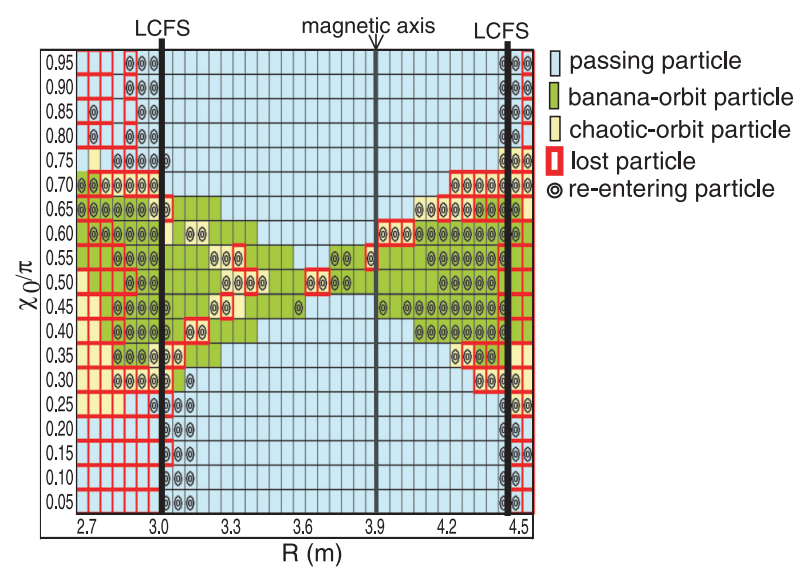

Fig. 4 Particle orbit classifications in magnetic field (ii) $\left(B_{\mathrm{ax}}=\right.$ $3 \mathrm{~T},\langle\beta\rangle=2.7 \%$, and $R_{\mathrm{ax}} \simeq 3.9 \mathrm{~m}$ ) in the space relating to the starting points versus the initial pitch angles.

the initial pitch angles. The initial conditions of the calculation shown in Fig. 4 are the same as those shown in Fig. 3, except for the averaged beta $\langle\beta\rangle$. The positions of the magnetic axis and the LCFS on the line of $Z=0$ on the horizontally elongated poloidal plane are also shown in Fig. 4. As shown in Fig. 1, the magnetic axis shifts from $R_{\mathrm{ax}}=3.6 \mathrm{~m}$ to $R_{\mathrm{ax}} \simeq 3.9 \mathrm{~m}$. The positions of the LCFS at both the inner and outer sides of the torus move to the plasma center. As a result, the minor radius of the closed flux surfaces on the line of $Z=0$ on the horizontally elongated poloidal plane in $\langle\beta\rangle=2.7 \%$ become smaller by about $20 \%$ compared to those in $\langle\beta\rangle=0 \%$. The center of the LCFS shifts in the direction of the major radius, since the position of the LCFS at the inner side of the torus shifts further than that at the outer side of the torus.

Comparing Fig. $4(\langle\beta\rangle=2.7 \%)$ with Fig. $3(\langle\beta\rangle=$ $0 \%$ ), the following differences can be observed. In $\langle\beta\rangle=$ $2.7 \%$, the number of chaotic-orbit particles decreases. Moreover, the number of lost particles traced from the inner side of the torus is larger than that in $\langle\beta\rangle=0 \%$ because of the large shift of the LCFS at the inner side of the torus in the direction of the major radius. Excluding these differences, there is no significant difference in the particle orbit classifications in the space relating to the starting points versus the initial pitch angles.

It is seen from Fig. $4(\langle\beta\rangle=2.7 \%)$ that particles traced from almost all starting points become re-entering particles. Here, we describe the re-entering particle traced from $3.6 \mathrm{~m} \leq R \leq 4.4 \mathrm{~m}$. In the case of $\langle\beta\rangle=0 \%$, only the chaotic-orbit particles become re-entering particles. In the case of $\langle\beta\rangle=2.7 \%$, not only chaotic-orbit particles but also banana-orbit particles become re-entering particles. As mentioned above, it should be noted that the bananaorbit particles form the closed drift surface.

We investigate the particles forming the closed drift surfaces, i.e., the passing and banana-orbit particles. Figure 5 shows the typical orbits of these particles in $\langle\beta\rangle=$

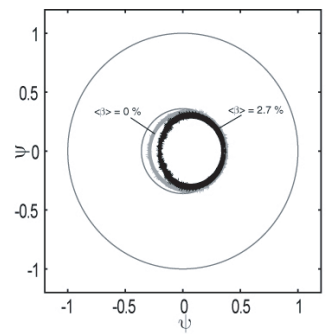

(a) passing particle $\left(\chi_{0}=0.1 \pi\right)$

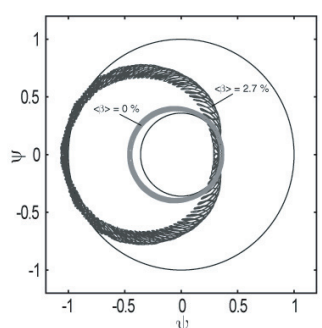

(b) banana orbit particle $\left(\chi_{0}=0.55 \pi\right)$
Fig. 5 Typical orbits of the passing and banana-orbit particles in $(\psi-\theta)$ coordinates. These particles are traced from the same starting points in $(\psi-\theta)$ coordinates. Black lines denote the orbits in the case of magnetic field (ii) $\left(B_{\mathrm{ax}}=\right.$ $3 \mathrm{~T},\langle\beta\rangle=2.7 \%$, and $R_{\mathrm{ax}} \simeq 3.9 \mathrm{~m}$ ) and gray lines denote the orbits in the case of magnetic field (i) $\left(B_{\mathrm{ax}}=3 \mathrm{~T}\right.$, $\langle\beta\rangle=0 \%$, and $\left.R_{\mathrm{ax}}=3.6 \mathrm{~m}\right)$. The flux surfaces at the starting point and the LCFS are shown for references.

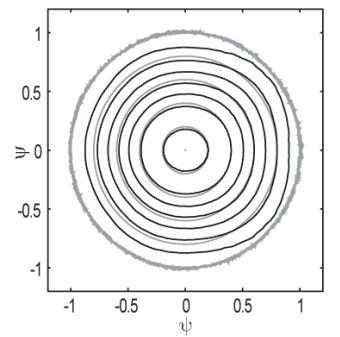

(a) magnetic field (i)

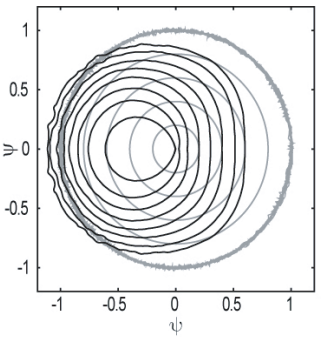

(b) magnetic field (ii)
Fig. 6 The cross-lines of $B=$ const. plane and $\boldsymbol{B} \cdot \nabla B=0$ plane with $(\boldsymbol{B} \cdot \nabla)^{2} B>0$ in the case of (a) magnetic field (i) ( $B_{\mathrm{ax}}=3 \mathrm{~T},\langle\beta\rangle=0 \%$, and $R_{\mathrm{ax}}=3.6 \mathrm{~m}$ ) and (b) magnetic field (ii) $\left(B_{\mathrm{ax}}=3 \mathrm{~T},\langle\beta\rangle=2.7 \%\right.$, and $\left.R_{\mathrm{ax}} \simeq 3.9 \mathrm{~m}\right)$. The flux surfaces are also shown as gray lines.

2.7\%. In $(\psi-\theta)$ coordinates, the orbit of the passing particle in $\langle\beta\rangle=2.7 \%$ almost agrees with that in $\langle\beta\rangle=0 \%$ (Fig. 5 (a)). In the rotating helical coordinate system, however, there is a significant difference in the orbits of the passing particles between $\langle\beta\rangle=0 \%$ and $\langle\beta\rangle=2.7 \%$. In contrast, the orbit of banana-orbit particles in $\langle\beta\rangle=2.7 \%$ significantly differs from that in $\langle\beta\rangle=0 \%$ (Fig. 5 (b)). In $\langle\beta\rangle=2.7 \%$, the banana-orbit particle moves across the flux surfaces and reaches the neighborhood of the LCFS. Therefore, most of the banana-orbit particles become reentering particles in the case of $\langle\beta\rangle=2.7 \%$. On the other hand, the banana-orbit particles in $\langle\beta\rangle=0 \%$ follow the orbit nearly concentrically with the flux surfaces in $(\psi-\theta)$ coordinates. Thus, there are no "re-entering banana-orbit particles" with the exception of the particles traced from the starting points near the LCFS.

In order to determine the cause of the difference in the banana orbits, in Fig.6, we draw the cross-lines of $B=$ const. plane and $\boldsymbol{B} \cdot \nabla B=0$ plane with $(\boldsymbol{B} \cdot \nabla)^{2} B>0$, which approximately give the orbits of deeply trapped particles, i.e., the banana-orbit particles [16]. It is noted that 
the cross-lines of $B=$ const. plane and $\boldsymbol{B} \cdot \nabla B=0$ plane with $(\boldsymbol{B} \cdot \nabla)^{2} B>0$ are almost the same as the $\bmod -B_{\min }$ contours in the Boozer coordinates. As shown in Fig. 6 (a), the cross-lines of $B=$ const. plane and $\boldsymbol{B} \cdot \nabla B=0$ plane with $(\boldsymbol{B} \cdot \nabla)^{2} B>0$ are concentric with the flux surfaces. In contrast, the cross-lines of $B=$ const. plane and $\boldsymbol{B} \cdot \nabla \boldsymbol{B}=0$ plane with $(\boldsymbol{B} \cdot \nabla)^{2} B>0$ in the finite beta plasma deviate from the flux surfaces (Fig. 6 (b)). The difference in the banana orbits shown in Fig. 5 (b) is attributed to this difference in the cross-lines of $B=$ const. plane and $\boldsymbol{B} \cdot \nabla B=0$ plane with $(\boldsymbol{B} \cdot \nabla)^{2} B>0$. The difference in the cross-lines of $B=$ const. plane and $\boldsymbol{B} \cdot \nabla \boldsymbol{B}=0$ plane with $(\boldsymbol{B} \cdot \nabla)^{2} B>0$ shown in Fig. 6 is caused because the distribution of the magnetic field strength in the finite beta plasmas, in which the structure of the flux surfaces varies significantly from $\langle\beta\rangle=0 \%$, rarely changes.

\subsection{Magnetic field (iii) $\left(B_{\mathrm{ax}}=0.5 \mathrm{~T},\langle\beta\rangle=\right.$ $2.7 \%$, and $R_{\mathrm{ax}} \simeq 3.9 \mathrm{~m}$ )}

We investigate the effects of the magnetic field strength on particle orbits by setting $B_{\mathrm{ax}}$ to $0.5 \mathrm{~T}$. As mentioned in Sec. $1, B_{\mathrm{ax}}=0.5 \mathrm{~T}$ is almost the same as the magnetic field strength used in high-beta experiments in the LHD [2]. Figure 7 shows the particle orbit classifications in magnetic field (iii) $\left(B_{\mathrm{ax}}=0.5 \mathrm{~T},\langle\beta\rangle=2.7 \%\right.$, and $R_{\mathrm{ax}} \simeq 3.9 \mathrm{~m}$ ) in the space relating to the starting points versus the initial pitch angles. The initial conditions of the calculation shown in Fig. 7 are the same as those shown in Fig. 4, except for the magnetic field strength. The positions of the magnetic axis and the LCFS are also shown.

In the particles traced from $3.1 \mathrm{~m} \leq R \leq 4.3 \mathrm{~m}$, the number of lost particles in $B_{\mathrm{ax}}=0.5 \mathrm{~T}$ is larger than that in $B_{\mathrm{ax}}=3 \mathrm{~T}$. In particular, most of the particles traced from the inner side of the torus with $\chi_{0}<0.5 \pi$ and those traced from the outer side of the torus with $\chi>0.5 \pi$ are lost, while such particles are confined in the case of $B_{\mathrm{ax}}=3 \mathrm{~T}$.

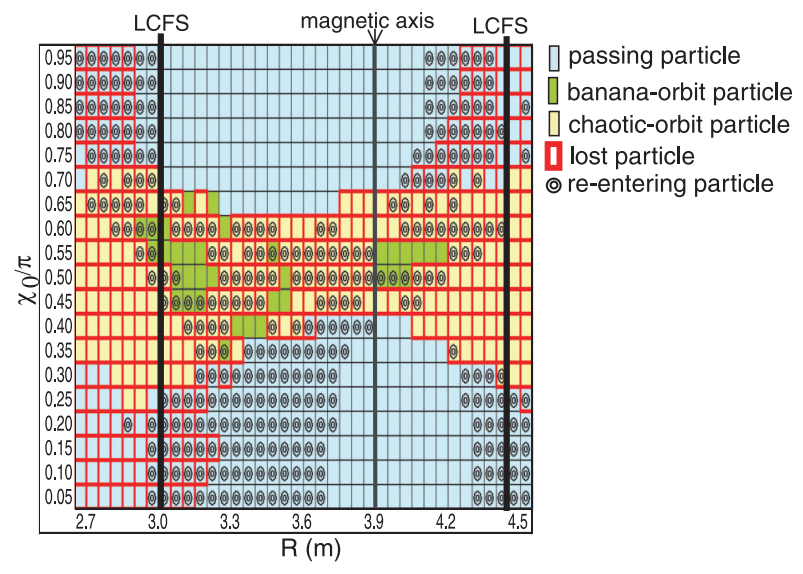

Fig. 7 Particle orbit classifications in magnetic field (iii) $\left(B_{\mathrm{ax}}=\right.$ $0.5 \mathrm{~T},\langle\beta\rangle=2.7 \%$, and $R_{\mathrm{ax}} \simeq 3.9 \mathrm{~m}$ ) in the space relating to the starting points versus the initial pitch angles.
The number of chaotic-orbit particles in $B_{\mathrm{ax}}=0.5 \mathrm{~T}$ increases compared with that in $B_{\mathrm{ax}}=3 \mathrm{~T}$. All of the chaotic-orbit particles in $B_{\mathrm{ax}}=0.5 \mathrm{~T}$ are lost within $30 \mathrm{~ms}$. Particles that have the same initial conditions as those of these chaotic-orbit particles are passing or banana-orbit particles and are confined within $30 \mathrm{~ms}$ in the case of $B_{\text {ax }}=3 \mathrm{~T}$.

In magnetic field (iii) $\left(B_{\mathrm{ax}}=0.5 \mathrm{~T},\langle\beta\rangle=2.7 \%\right.$, and $R_{\mathrm{ax}} \simeq 3.9 \mathrm{~m}$ ), the re-entering particles exist in the particles traced from almost all starting points independent of particle orbit classifications. In particular, regarding the particles traced from $3.0 \mathrm{~m} \leq R \leq 3.8 \mathrm{~m}$ with $\chi_{0}<0.5 \pi$, almost all the passing particles are re-entering particles in magnetic field (iii) $\left(B_{\mathrm{ax}}=0.5 \mathrm{~T},\langle\beta\rangle=2.7 \%\right.$, and $\left.R_{\mathrm{ax}} \simeq 3.9 \mathrm{~m}\right)$. In magnetic field (ii) $\left(B_{\mathrm{ax}}=3 \mathrm{~T},\langle\beta\rangle=2.7 \%\right.$, and $R_{\mathrm{ax}} \simeq 3.9 \mathrm{~m}$ ), no re-entering particle exists as passing particles traced from $3.3 \mathrm{~m} \leq R \leq 4.3 \mathrm{~m}$.

We also investigate the particles forming the closed drift surfaces in magnetic field (iii) $\left(B_{\mathrm{ax}}=0.5 \mathrm{~T},\langle\beta\rangle=\right.$ $2.7 \%$, and $R_{\mathrm{ax}} \simeq 3.9 \mathrm{~m}$ ). Figure 8 shows the typical orbit of these particles. Compared with the orbit of the passing particle in $B_{\mathrm{ax}}=3 \mathrm{~T}$, the deviation of the orbit of the passing particle in $B_{\mathrm{ax}}=0.5 \mathrm{~T}$ from the flux surfaces is large. In order to examine the cause of this large deviation of the orbit of the passing particle in $B_{\mathrm{ax}}=0.5 \mathrm{~T}$, we estimate the $\nabla B$ drift velocities $\left(V_{\nabla B}\right)$ at the starting points. In $B_{\mathrm{ax}}=0.5 \mathrm{~T}, V_{\nabla B}=1.4 \times 10^{4} \mathrm{~m} / \mathrm{s}$, and in $B_{\mathrm{ax}}=3 \mathrm{~T}$, $V_{\nabla B}=0.23 \times 10^{4} \mathrm{~m} / \mathrm{s} . \quad V_{\nabla B}$ in $B_{\mathrm{ax}}=0.5 \mathrm{~T}$ is about six times larger than that in $B_{\mathrm{ax}}=3 \mathrm{~T}$. Because the deviation of the orbit of the passing particle from the flux surfaces is approximately proportional to $V_{\nabla B}$, the deviation of the orbit of the passing particle in $B_{\mathrm{ax}}=0.5 \mathrm{~T}$ from the flux surfaces is larger than that in $B_{\mathrm{ax}}=3 \mathrm{~T}$. The orbit of the passing particle in $B_{\mathrm{ax}}=0.5 \mathrm{~T}$ has a wide minor radial width, which will be discussed in Sec. 4 .

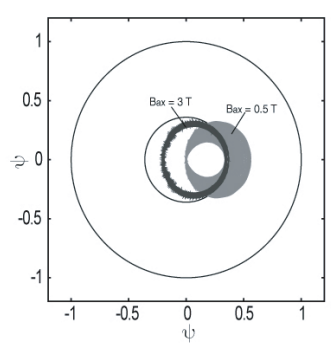

(a) passing particle $\left(\chi_{0}=0.1 \pi\right)$

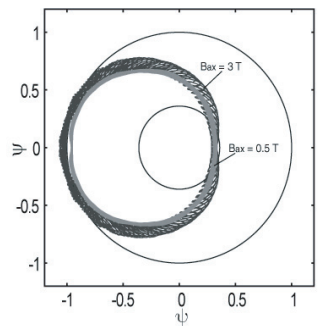

(b) banana orbit particle $\left(\chi_{0}=0.55 \pi\right)$
Fig. 8 Typical orbits of the passing and banana-orbit particles in magnetic field (iii) $\left(B_{\mathrm{ax}}=0.5 \mathrm{~T},\langle\beta\rangle=2.7 \%\right.$, and $R_{\mathrm{ax}} \simeq$ $3.9 \mathrm{~m})$ in $(\psi-\theta)$ coordinates. These particles are traced from the same starting points in $(\psi-\theta)$ coordinates. Black lines denote the orbits in the case of magnetic field (ii) $\left(B_{\mathrm{ax}}=3 \mathrm{~T},\langle\beta\rangle=2.7 \%\right.$, and $\left.R_{\mathrm{ax}} \simeq 3.9 \mathrm{~m}\right)$ and gray lines the orbits in the case of the magnetic field (iii) $\left(B_{\mathrm{ax}}=\right.$ $0.5 \mathrm{~T},\langle\beta\rangle=2.7 \%$, and $R_{\mathrm{ax}} \simeq 3.9 \mathrm{~m}$ ). The flux surfaces at the starting point and the LCFS are shown for reference. 


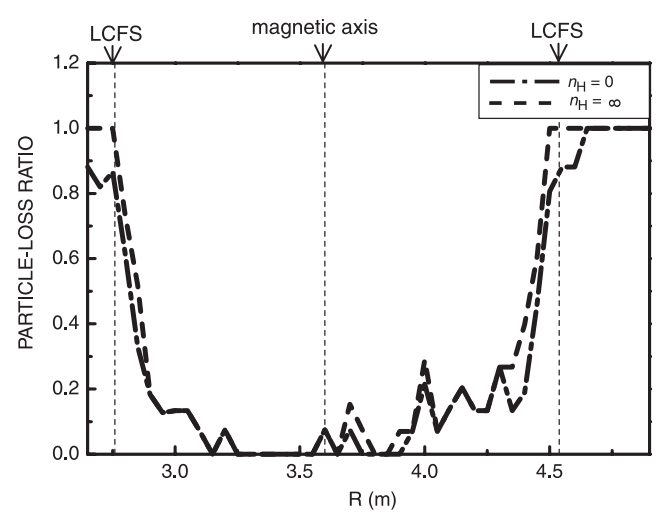

(a) magnetic field (i) $\left(n_{\mathrm{H}}=0\right.$ and $\left.\infty\right)$

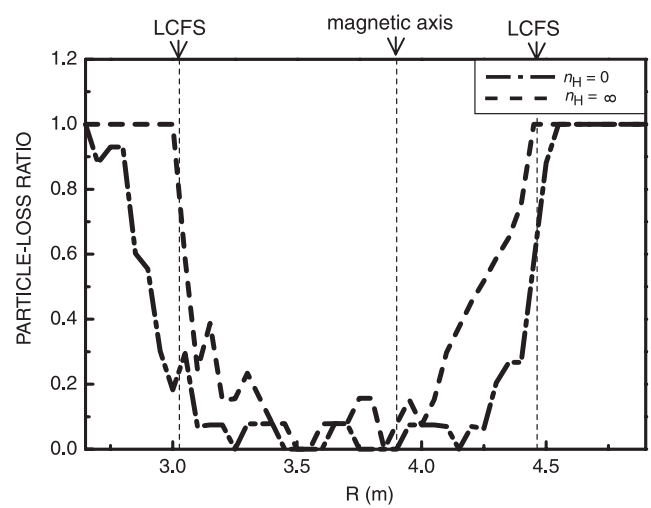

(c) magnetic field (ii) $\left(n_{H}=0\right.$ and $\left.\infty\right)$

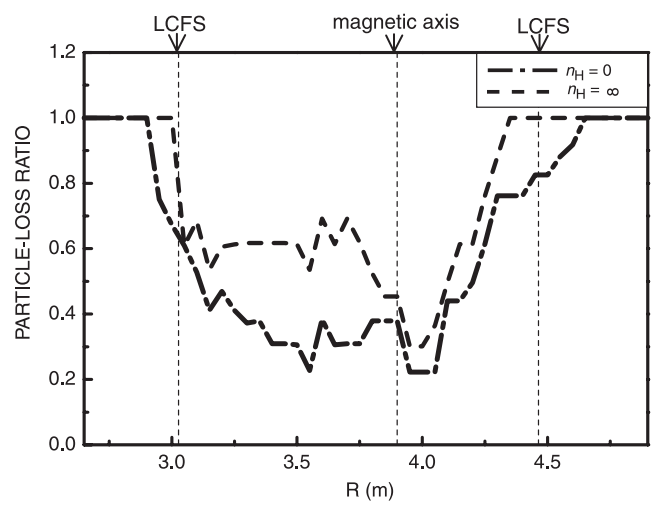

(e) magnetic field (iii) $\left(n_{\mathrm{H}}=0\right.$ and $\left.\infty\right)$

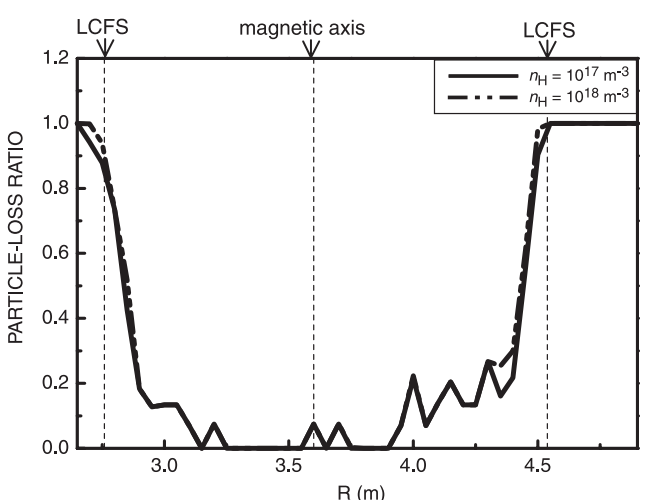

(b) magnetic field (i) $\left(n_{\mathrm{H}}=10^{17}\right.$ and $\left.10^{18} \mathrm{~m}^{-3}\right)$

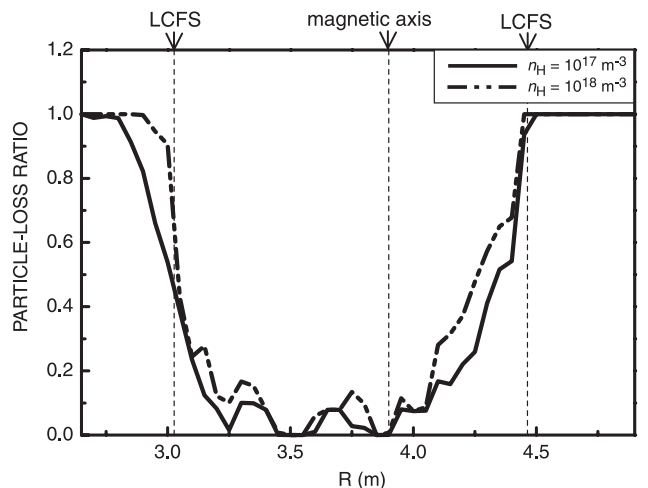

(d) magnetic field (ii) $\left(n_{\mathrm{H}}=10^{17}\right.$ and $\left.10^{18} \mathrm{~m}^{-3}\right)$

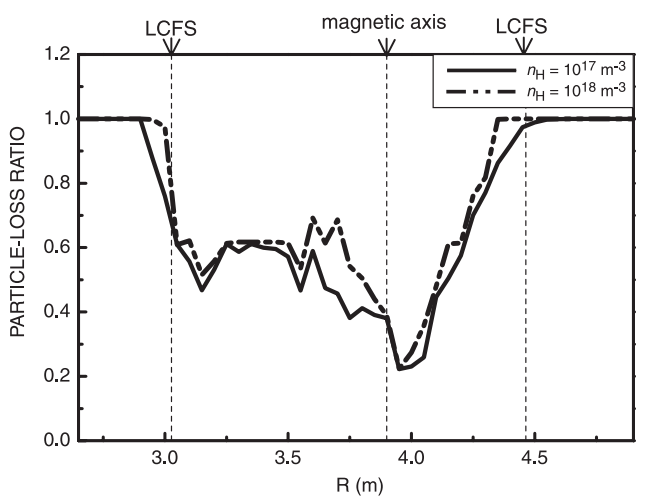

(f) magnetic field (iii) $\left(n_{\mathrm{H}}=10^{17}\right.$ and $\left.10^{18} \mathrm{~m}^{-3}\right)$

Fig. 9 Particle-loss ratio after $30 \mathrm{~ms}$ particle tracing. The horizontal axis is the major radius of the starting points set on the line of $Z=0$ on the horizontally elongated poloidal plane. The vertical axis denotes the particle-loss ratio averaged over the pitch angles at each starting point. The positions of the magnetic axis and the LCFS on the line of $Z=0$ on the horizontally elongated poloidal plane are also shown.

It is seen from Fig. 8 (b) that the banana orbit in $B_{\mathrm{ax}}=$ $0.5 \mathrm{~T}$ almost agrees with that in $B_{\mathrm{ax}}=3 \mathrm{~T}$. This is because the shapes of the cross-lines of $B=$ const. plane and $\boldsymbol{B}$. $\nabla B=0$ plane with $(\boldsymbol{B} \cdot \nabla)^{2} B>0$ are independent of the magnetic field strength.

\subsection{Effects of re-entering particles on parti- cle confinement}

In order to investigate the effects of the re-entering particles on particle confinement, we evaluate the particleloss ratio averaged over pitch angles at each starting points [14] in all the magnetic fields. We also investigate the effect of the charge-exchange reaction on the re-entering particles. Thus, the following assumptions are made. The charge-exchange reaction occurs only with the hydrogen atoms. The density of hydrogen atoms $\left(n_{\mathrm{H}}\right)$ is uniform outside the LCFS and $n_{\mathrm{H}}=0$ inside the LCFS. After $J$-th time re-entering, the existing probability of the proton is 
approximately given as

$$
\alpha_{J}=\exp \left(-\frac{1}{\lambda}\left(\sum_{j=1}^{\mathrm{J}} s_{j}\right)\right),
$$

where $s_{j}$ is the path length of $j$-th time re-entering outside the LCFS, and $\lambda$ is the mean free path of the chargeexchange reaction determined by $\lambda=1 / n_{\mathrm{H}} \sigma_{\mathrm{H}}$. Here, $\sigma_{\mathrm{H}}$ is the cross-section of the charge-exchange reaction between a proton and a hydrogen atom. When the energy of the proton is $100 \mathrm{keV}, \sigma_{\mathrm{H}}=9.71 \times 10^{-22} \mathrm{~m}^{2}$ [17]. Figure 9 shows the particle-loss ratio after tracing particles for $30 \mathrm{~ms}$. Figures 9 (a) and (b) show the results in magnetic field (i), Figs. 9 (c) and (d) in magnetic field (ii), and Figs. 9 (e) and (f) in magnetic field (iii). In Fig. 9, the dashed-dotted lines correspond to the $n_{\mathrm{H}}=0$ case, which does not show any particle loss due to the charge-exchange reaction. The dashed lines correspond to the $n_{\mathrm{H}}=\infty$ case, in which the re-entering particles are regarded as lost particles. The solid lines denote the $n_{\mathrm{H}}=10^{17} \mathrm{~m}^{-3}$ and the dashed twodotted lines denote $n_{\mathrm{H}}=10^{18} \mathrm{~m}^{-3}$. The positions of the magnetic axis and the LCFS on the line of $Z=0$ on the horizontally elongated poloidal plane are also shown.

It is seen from Fig. 9 (a) that the particle-loss ratio in the case of $n_{\mathrm{H}}=0$ (dashed-dotted line) is lower than that in the case of $n_{\mathrm{H}}=\infty$ (dashed line). This implies that reentering particles exist in the vacuum magnetic field. Comparing Figs. 9 (c) and (e) with Fig. 9 (a), the differences in the particle-loss ratio between $n_{\mathrm{H}}=0$ and $n_{\mathrm{H}}=\infty$ cases is remarkable in $\langle\beta\rangle=2.7 \%$. This shows that the number of re-entering particles in the finite beta plasma is larger than that in the vacuum magnetic field. Therefore, the role of the re-entering particles in the finite beta plasmas of the LHD is more important than that in the vacuum magnetic field. The particle-loss ratios in the $n_{\mathrm{H}}=\infty$ cases (dashed lines in Figs. 9 (a), (c), and (e)) are the same as those in the particle orbit analysis using the Boozer coordinates, in which the particle-loss boundary is set on the LCFS. Thus, the difference in the particle-loss ratios between $n_{\mathrm{H}}=0$ and $n_{\mathrm{H}}=\infty$ cases (Figs. 9 (a), (c), and (e)) implies that the number of lost particles is overestimated in the particle orbit analysis using the Boozer coordinates.

Next, the particle-loss ratios when the particle loss due to the charge-exchange reaction is ignored $\left(n_{\mathrm{H}}=0\right)$ are discussed. It is seen from Figs. 9 (a) and (c) that there is no significant difference in the dashed-dotted lines $\left(n_{\mathrm{H}}=0\right)$ between the $\langle\beta\rangle=0 \%$ and $\langle\beta\rangle=2.7 \%$ cases. Thus, most particles confined in the case of $\langle\beta\rangle=0 \%$ are also confined in the case of $\langle\beta\rangle=2.7 \%$ when the particle loss due to the charge-exchange reaction is ignored. On the other hand, the particle-loss ratio in $B_{\mathrm{ax}}=0.5 \mathrm{~T}$ is higher than that in $B_{\mathrm{ax}}=3 \mathrm{~T}$. This is because the number of lost particles in $B_{\mathrm{ax}}=0.5 \mathrm{~T}$ is larger than that in $B_{\mathrm{ax}}=3 \mathrm{~T}$ (Fig. 7).

Independent of the magnetic field, the particle-loss ratio in the case of $n_{\mathrm{H}}=10^{18} \mathrm{~m}^{-3}$ are almost the same as that in the case of $n_{\mathrm{H}}=\infty$. In the case of $n_{\mathrm{H}}=\infty$, there are no re-entering particles. On the other hand, in the case of $n_{\mathrm{H}}=10^{18} \mathrm{~m}^{-3}$, the lost particles due to the chargeexchange can pass in and out of the LCFS (re-enter) many times. The particle-loss ratio in the case of $n_{\mathrm{H}}=10^{17} \mathrm{~m}^{-3}$ is a little lower than that in the case of $n_{\mathrm{H}}=10^{18} \mathrm{~m}^{-3}$. These results imply that the loss of re-entering particles due to the charge-exchange reaction depends on the density of neutral particles in the periphery.

In magnetic field (i) $\left(B_{\mathrm{ax}}=3 \mathrm{~T},\langle\beta\rangle=0 \%\right.$, and $\left.R_{\mathrm{ax}}=3.6 \mathrm{~m}\right)$, the particle-loss ratios in the $n_{\mathrm{H}}=10^{18} \mathrm{~m}^{-3}$ and $n_{\mathrm{H}}=10^{17} \mathrm{~m}^{-3}$ cases are almost the same as that in the $n_{\mathrm{H}}=0$ case (Figs. 9 (a) and (b)). This implies that most of the re-entering particles consist of "lost" chaotic-orbit particles without a particle loss cased by the charge-exchange reaction in magnetic field (i) $\left(B_{\mathrm{ax}}=3 \mathrm{~T},\langle\beta\rangle=0 \%\right.$, and $\left.R_{\mathrm{ax}}=3.6 \mathrm{~m}\right)$. Thus, the charge-exchange reaction in magnetic field (i) $\left(B_{\mathrm{ax}}=3 \mathrm{~T},\langle\beta\rangle=0 \%\right.$, and $\left.R_{\mathrm{ax}}=3.6 \mathrm{~m}\right)$ rarely affects particle confinement. On the other hand, in magnetic fields (ii) and (iii) (in the case of $\langle\beta\rangle=2.7 \%$ ), the particle-loss ratios in the $n_{\mathrm{H}}=10^{18} \mathrm{~m}^{-3}$ and $n_{\mathrm{H}}=10^{17} \mathrm{~m}^{-3}$ cases are higher than that in the $n_{\mathrm{H}}=0$ case (Figs. 9 (c)(f)).

\section{Discussion}

We can clarify the cause of the decrease in the number of chaotic-orbit particles when the beta value increases. Figure 10 shows the variation of the magnetic field strength along the magnetic field line traced from $R=4.1 \mathrm{~m}$ with $v_{\|}>0$. The horizontal axis is the connection length of the magnetic field line. The vertical axis denotes $\widehat{B}=B / B_{\text {start }}$. Here, $B_{\text {start }}$ is the magnetic field strength at the starting point. It is seen that the toroidal ripple in the case of $\langle\beta\rangle=2.7 \%$ is shallower than that in the case of $\langle\beta\rangle=0 \%$. This implies that some particles trapped by toroidal ripples

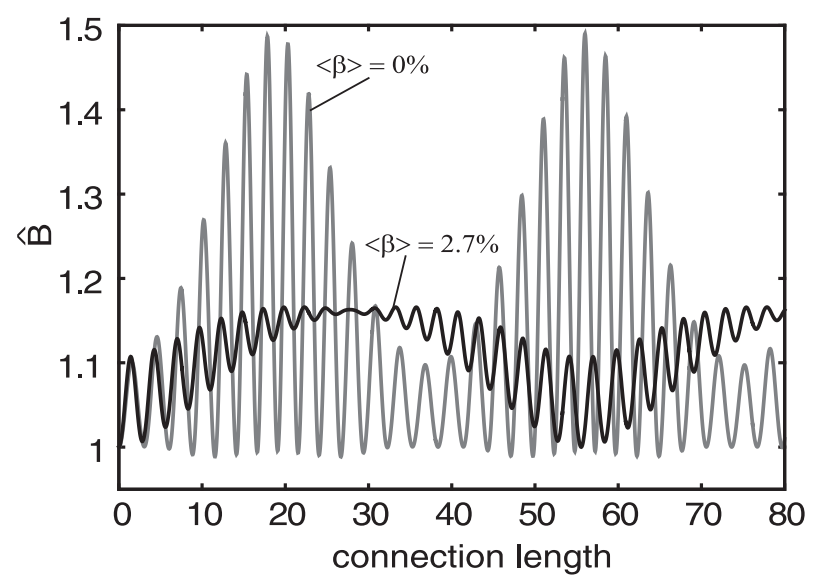

Fig. 10 Variation of the magnetic field strength along the magnetic field line traced from $R=4.1 \mathrm{~m}$ with $v_{\|}>0$. The horizontal axis is the connection length of the magnetic field line. The vertical axis denotes $\widehat{B}=B / B_{\text {start }}$. Here, $B_{\text {start }}$ is the magnetic field strength at the starting point. 
in the case of $\langle\beta\rangle=0 \%$ cannot be trapped in the case of $\langle\beta\rangle=2.7 \%$. Such particles become passing particles when $\langle\beta\rangle=2.7 \%$. Therefore, the number of chaotic-orbit particles, which repeatedly transit between the localized orbit and the blocked orbit, decreases when $\langle\beta\rangle=2.7 \%$. As a result, the number of passing particles increases when $\langle\beta\rangle=2.7 \%$.

Next, we investigate the cause of the increase in the number of lost chaotic-orbit particles in $B_{\mathrm{ax}}=0.5 \mathrm{~T}$. Figure 11 shows the typical orbits of the chaotic orbit particles traced from $R=4.1 \mathrm{~m}$ in two magnetic fields $\left(B_{\mathrm{ax}}=3 \mathrm{~T}\right.$, $\langle\beta\rangle=0 \%$ and $\left.B_{\mathrm{ax}}=2 \mathrm{~T},\langle\beta\rangle=0 \%\right)$. The orbit of the chaotic-orbit particle in the case of $B_{\mathrm{ax}}=2 \mathrm{~T}$ is widespread compared with that in the case of $B_{\mathrm{ax}}=3 \mathrm{~T}$. Therefore, the chaotic-orbit particles in a weak magnetic field (as in $B_{\mathrm{ax}}=0.5 \mathrm{~T}$ ) easily reach the region outside the LCFS or the chaotic field line region. Because the connection lengths are short in such a region, most chaotic-orbit particles are lost in $B_{\mathrm{ax}}=0.5 \mathrm{~T}$.

We investigate the minor radial width of a passing particle shown in Fig. 8. Particles are drifted by $\nabla_{\theta} B$ with the velocity $\left(v_{\mathrm{D}}^{\theta}\right)$ during poloidal rotations. Figure 12 shows the directions of $v_{\mathrm{D}}^{\theta}$ and the typical orbit of a passing particle in $B_{\mathrm{ax}}=0.5 \mathrm{~T}$. These particles alternately move to the outer and inner sides in the minor radius direction because of $v_{\mathrm{D}}^{\theta}$ (Fig. 12 (a)). As a result, the passing particles bounce between the inside and outside of the flux surface, as shown in Fig. 12 (b). The diameter of this circular motion is the minor radial width of the passing particles. The

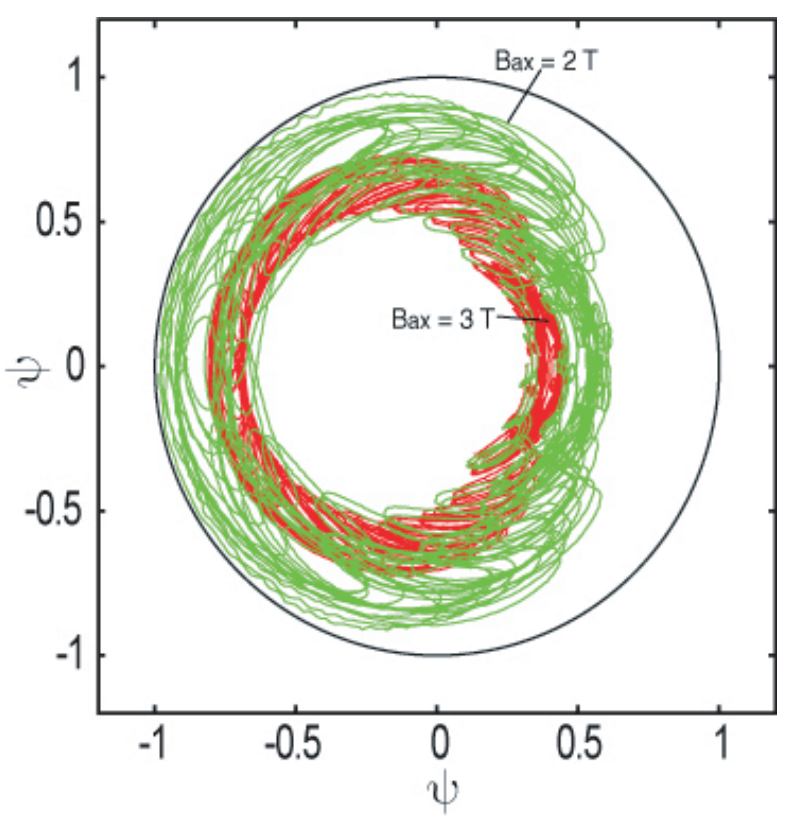

Fig. 11 Typical orbits of the chaotic-orbit particles traced from $R=4.1 \mathrm{~m}$ in two magnetic fields: $B_{\mathrm{ax}}=3 \mathrm{~T},\langle\beta\rangle=0 \%$ (red) and $B_{\mathrm{ax}}=2 \mathrm{~T},\langle\beta\rangle=0 \%$ (green). The LCFS is shown for references. minor radial width is approximately given by

$$
\begin{aligned}
\Delta_{\mathrm{D}}^{\theta} & \sim\left|\boldsymbol{v}_{\mathrm{D}}^{\theta}\right| \times\left|\frac{(\pi / 10) R_{0}}{\boldsymbol{v}_{\|}}\right| \\
& =M\left|\left(\frac{\boldsymbol{v}_{\perp}^{2}}{2}\right) \frac{\boldsymbol{B} \times \nabla_{\theta} B}{q \boldsymbol{B}^{3}}\right| \times\left|\frac{(\pi / 10) R_{0}}{\boldsymbol{v}_{\|}}\right|,
\end{aligned}
$$

where $v_{\perp}$ and $v_{\|}$are the velocity perpendicular and parallel to the magnetic field line, $M$ is the particle mass, and $q$ is the electric charge. Here, it is assumed that the $\left|\boldsymbol{v}_{\mathrm{D}}^{\theta}\right|$ of the passing particle is constant when the passing particle moves from $\phi=0$ to $\phi=0.1 \pi$ (a half helical pitch). In the case of $\nabla_{\theta} B / B=0.5 \mathrm{~m}^{-1}$, the minor radial width $\Delta_{\mathrm{D}}^{\theta}$ becomes $1.2 \times 10^{-2} \mathrm{~m}\left(B_{\mathrm{ax}}=0.5 \mathrm{~T}\right)$ and $0.2 \times 10^{-2} \mathrm{~m}$ $\left(B_{\mathrm{ax}}=3 \mathrm{~T}\right)$. These values are smaller than the averaged minor radius of the LHD.

In this paper, the effects of collisions between particles are not considered. Of course, collisions have significant effects on the orbit of a particle, particularly on a re-entering particle in the finite beta plasma. A particle orbit analysis that includes Coulomb collisions is planned for the future.

As mentioned above, the loss of the re-entering particles due to the charge-exchange reaction depends on the density of the neutral particles in the periphery. In order to trace the re-entering particles more precisely, a study using an accurate distribution of the density of the neutrals is required. The energy of the particles also affects the chargeexchange reaction rate. A calculation that includes energy scattering is therefore needed.

In this study, $100 \mathrm{keV}$ proton orbits are investigated by numerically solving the guiding-center equations. The Larmor radius of $100 \mathrm{keV}$ protons in $B_{\mathrm{ax}}=3 \mathrm{~T}$ is about $0.015 \mathrm{~m}$. On the other hand, the Larmor radius of $100 \mathrm{keV}$ protons in $B_{\mathrm{ax}}=0.5 \mathrm{~T}$ is about $0.091 \mathrm{~m}$, i.e., about a seventh of the averaged plasma minor radius $\left(a_{\mathrm{p}}=0.64 \mathrm{~m}\right)$. In $B_{\mathrm{ax}}=0.5 \mathrm{~T}$, the $100 \mathrm{keV}$ proton could not be accurately traced by solving the guiding-center equations. We will investigate the particle orbits by numerically solving the equation of motion in the near future.

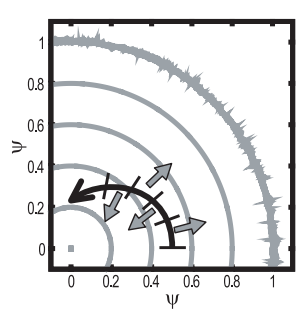

(a) directions of $v_{\mathrm{D}}^{\theta}$

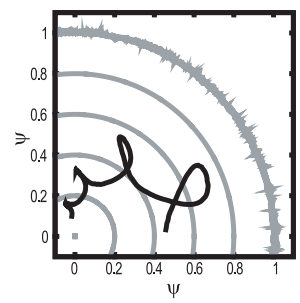

(b) passing partcile $\left(B_{\mathrm{ax}}=0.5 \mathrm{~T}\right)$
Fig. 12 Directions of $v_{\mathrm{D}}^{\theta}$ and the typical orbit of a passing particle in magnetic field (iii) $\left(B_{\mathrm{ax}}=0.5 \mathrm{~T},\langle\beta\rangle=2.7 \%\right.$, and $R_{\mathrm{ax}} \simeq$ $3.9 \mathrm{~m}$ ). (a) Directions of $\boldsymbol{v}_{\mathrm{D}}^{\theta}$ in the model orbit of a passing particle. The black arrow shows the model orbit, and the gray arrows show the directions of $\boldsymbol{v}_{\mathrm{D}}^{\theta}$. (b) Typical orbit of a passing particle in magnetic field (iii). 


\section{Conclusion}

By numerically solving the guiding-center equations, we have traced high-energy particles in an equilibrium magnetic field in a finite beta plasma of the LHD calculated using HINT. We have appropriately treated re-entering particles by setting the particle-loss boundary on the vacuum vessel wall. We have investigated the difference in the particle orbits caused by the volume-averaged beta value and/or the magnetic field strength. In addition, we have investigated the effects of the charge-exchange reaction on the re-entering particles. The following results have been obtained.

There is no significant difference in the particle orbit classifications in the space relating to the starting points versus the initial pitch angles between $\langle\beta\rangle=0 \%$ and $\langle\beta\rangle=2.7 \%$ in the cases of $B_{\mathrm{ax}}=3 \mathrm{~T}$. The deviation of the orbit of a passing particle from the flux surface is independent of the beta value. In contrast, the deviation of the orbit of a banana-orbit particle from the flux surface strongly depends on the beta value. This is because the distribution of the magnetic field strength in finite beta plasmas, in which the structure of the flux surfaces significantly changes from the vacuum magnetic field, rarely changes.

The number of chaotic-orbit particles in $B_{\mathrm{ax}}=0.5 \mathrm{~T}$ increases compared with that in $B_{\mathrm{ax}}=3 \mathrm{~T}$, and all chaoticorbit particles are lost within $30 \mathrm{~ms}$. When changing the magnetic field from $B_{\mathrm{ax}}=3 \mathrm{~T}$ to $B_{\mathrm{ax}}=0.5 \mathrm{~T}$, the deviation of the orbit of a passing particle becomes large. It is also found that the drift caused by $\nabla_{\theta} B$ determines the minor radial width of the passing particles. On the other hand, a banana orbit in $B_{\mathrm{ax}}=0.5 \mathrm{~T}$ almost agrees with that in $B_{\mathrm{ax}}=3 \mathrm{~T}$. This is because the shapes of the cross-lines of $B=$ const. plane and $\boldsymbol{B} \cdot \nabla B=0$ plane with $(\boldsymbol{B} \cdot \nabla)^{2} B>0$ are independent of the magnetic field strength.

Re-entering particles, whose existence has been shown in the vacuum magnetic field, are also found to exist in the finite beta plasma independent of the magnetic field strength. The number of re-entering particles in the finite beta plasma is larger than that in the vacuum magnetic field. Particles traced from almost all starting points can be re-entering particles in the finite beta plasma. Therefore, the role of re-entering particles in the finite beta plasmas of the LHD is more important than that in the vacuum magnetic field. When the peripheral neutral density is small $\left(n_{\mathrm{H}} \ll 10^{17} \mathrm{~m}^{-3}\right)$, re-entering particles play more important roles in processes such as plasma heating.

It is confirmed that the loss of re-entering particles due to the charge-exchange reaction depends on the density of neutrals in the periphery. Although the charge-exchange reaction rarely affects particle confinement in the vacuum magnetic field, the effect of the charge-exchange reaction on particle confinement in the finite beta plasma is large.

\section{Acknowledgements}

We thank Prof. S. Oikawa (Hokkaido University) for fruitful discussions. We are grateful to Prof. T. Watanabe (National Institute for Fusion Science (NIFS)) for his useful advice. This work is supported by the collaborative research program of NIFS (NIFS06KLHH302) and is partially supported by a Grant-in-Aid for Scientific Research (B) (18360445) of the Japan Society for the Promotion of Science (JSPS).

[1] A. Iiyosi et al., Nucl. Fusion 39, 1245 (1999).

[2] O. Motojima et al., Proc. 21th Fusion Energy conf. OV/2-1 (chengdu, 2007).

[3] T. Hayashi et al., Contrib. Plasma Phys. 42, 2-4 309 (2002).

[4] A.H. Boozer, Phys. Fluids 23, 904 (1980).

[5] S. Murakami, J. Plasma Fusion Res. 80, 725 (2004).

[6] R.H. Fowler, R.N. Morris, J.A. Rome and K. Hanatani, Nucl. Fusion 30, 997 (1990).

[7] K. Hanatani and F.-P. Penningsfeld, Nucl. Fusion 32, 1769 (1992).

[8] T. Watanabe, A. Ishida and T. Hatori, Kakuyugo Kenkyu 68, 298 (1992).

[9] T. Hayashi, Theory of Fusion Plasmas, EUR 12149 EN 11 (1989).

[10] K. Harafuji, T. Hayashi and T. Sato, J. Comput. Phys. 81, 169 (1989).

[11] T. Watanabe and H. Akao, J. Plasma Fusion. Res. 73, 186 (1997).

[12] D. Sarafyan, J. Math, Anal and Appl. 40, 436 (1972).

[13] T. Watanabe, Trans. Jpn. Soc. Ind. Appl. Mat. 1, 101 (1991) [in Japanese].

[14] Y. Matsumoto, T. Nagaura, S. Oikawa and T. Watanabe, Jpn. J. Appl. Phys. 43, 332 (2004).

[15] A. Gibson and J.B. Taylor, Phys. Fluids 10, 2653 (1967).

[16] Y. Matsumoto, S. Oikawa and T. Watanabe, J. Phys. Soc. Jpn. 71, 1684 (2002).

[17] A.C. Riviere, Nucl. Fusion 11, 363 (1971). 